\title{
A model for the construction of the network in phosphate glasses through viscosity-structure relationships
}

Francisco Muñoz*, Laura Muñoz-Senovilla

Ceramics and Glass Institute (CSIC), Madrid (Spain)

\begin{abstract}
We have established a relationship between the viscosity activation energy of metaphosphate melts and the NMR chemical shift anisotropy of phosphorus that is measured in their corresponding glasses, and proposed a model by which the fragility of the glasses can be explained as dependent on the particular modified random network that is originated upon cooling. The increase of the modifier field strength gives rise to higher viscosity activation energy during the glass formation upon cooling, with a maximum at the calcium metaphosphate composition. The lower rates of increase of viscosity would produce network structures characterized by higher anisotropicity and be the responsible for the higher fragility of the glasses, which can be defined as a measure of the degree of difference between the structures of the glassy state and the melt from which this is obtained.
\end{abstract}

Keywords: Phosphate Glasses; Viscosity; Glass Structure; NMR; Fragility

*Corresponding author: fmunoz@icv.csic.es

Instituto de Cerámica y Vidrio (CSIC), Kelsen 5, 28049 Madrid (Spain) 


\section{Introduction}

In a previous work, we published a study on the determination of the fragility from the viscosity-temperature curves of a series of metaphosphate glasses [1]. We made therein an analysis of the influence of the model employed to fit the viscosity data points on the obtained fragility values, and we calculated the activation energy of viscosity at the high and low viscosity ranges. In our conclusions, we pointed out to the fact that, showing different behaviors, the activation energies obtained in both parts of the viscosity-temperature curves should be influenced by different factors. Basically, the activation energy of viscosity above the glass transition would be determined not only by the overall strength of the glass network through the different field strength of the modifiers, but also by the medium range order structure of the phosphate network at each particular composition. However, the activation energy of viscosity for the phosphate melts showed a regular increasing tendency with the cationic potential of the modifier cation.

We did also analyze the structure of the glasses through Raman and Nuclear Magnetic Resonance (NMR) spectroscopies and found similar features as gathered in previous literature [2]. For instance, the isotropic chemical shift of ${ }^{31} \mathrm{P}$ nuclei moves linearly up-field with the cationic potential of the modifier cation [1,3]; however, we observed that the chemical shift anisotropy (CSA) of phosphorus does not vary linearly with the composition. The last increases first from $\mathrm{Na}$ to $\mathrm{Ca}$ metaphosphates, then decreases for $\mathrm{Zn}$ and $\mathrm{Mg}$ glasses. In a first approximation we interpreted the variation of this CSA as a means to characterize the different configurations in which the phosphate tetrahedral units might arrange, in either rings or chains, as it was also proposed by other authors $[4,5]$. The glasses showing a higher CSA would be formed by a bigger number of rings ( $\mathrm{Na}, \mathrm{Li}, \mathrm{Ba})$, while those with lower CSA numbers would have an increased number of chains ( $\mathrm{Sr}, \mathrm{Ca}, \mathrm{Zn}, \mathrm{Mg}$ ). Despite these efforts, we did not find further clear correlation between the viscosity activation energy in the high 
and low ranges with the medium range order structure of the $\mathrm{PO}_{4}$ tetrahedral units, but in the work by Muñoz-Senovilla et al. we also plotted the ratio of Doremus between the activation energies at the high and low viscosity ranges and pointed out that some sort of relationship should exist between the first and the chemical shift anisotropy, where lower degrees of anisotropy may imply lower values of the Doremus ratio, i.e. lower fragility values.

In the present work, we have used the measured CSA values of ${ }^{31} \mathrm{P}$ from NMR data, and the viscosity activation energy of the metaphosphate melts, to propose a model to explain the way through which the melt structure rearrange itself upon cooling giving rise to particular modified random glass network that may serve to interpret the structure-properties relationships.

\section{Experimental}

The glasses studied in this work are of the metaphosphate composition of alkali, alkalineearth and zinc modifiers, which viscosity data have been taken from the previous work by the authors $[1,6]$. The batches were obtained from mixing reagent grade raw materials that were melted during $2 \mathrm{~h}$ at temperatures ranging from $900^{\circ} \mathrm{C}$ to $1200^{\circ} \mathrm{C}$ depending on their composition. Viscosity measurements were performed through the beam-bending and rotation methods at the high and low viscosity ranges and according to the ISO 7884-2 and ISO-7884-4 standard testing conditions, respectively, as also described in [1,6]. Viscosity data between $10^{3}-10^{1} \mathrm{dPa}$.s were determined using rotation speeds of 3 to $15 \mathrm{rpm}$ for $15 \mathrm{~min}$ and up to 3 data were taken at each temperature. On the other hand, weights of 10 to $200 \mathrm{~g}$ were employed in the viscosity range $10^{12.5}-10^{9} \mathrm{dPa}$.s for the beam-bending mode. The chemical shift anisotropy values of ${ }^{31} \mathrm{P}$ have been taken from the simulation of the static ${ }^{31} \mathrm{P}$ NMR spectra as obtained in a BRUKER ASX 400 spectrometer [1,6]. 


\section{Results}

The viscosity activation energy of the phosphate melts $\left(E_{a}\right.$ low $\left.\eta\right)$, i.e. low viscosity range, that was measured by Muñoz-Senovilla et al. in [1], has been represented in Fig. 1 as a function of the ionic field strength of the modifier cation (IFS) [7]. It is assumed that at the temperatures of the liquids in equilibrium, the entropy term of the free activation energy becomes approximately zero and the apparent activation energy that is determined from the slope of the Arrhenius plot of the viscosity data against inverse temperature can be equivalent to the free activation energy in the low viscosity range [8]. Figure 1 shows that the $\mathrm{E}_{\mathrm{a}}$ of the viscous flow of the melts increases with the modifier field strength up to the $\mathrm{Ca}\left(\mathrm{PO}_{3}\right)_{2}$ composition, then it decreases for $\mathrm{Mg}$ and $\mathrm{Zn}$ metaphosphates. In both parts of the curve, below and above the IFS value for $\mathrm{Ca}$, there seems to be a linear variation of the activation energy; however, even though zinc and magnesium possess higher field strength than calcium the corresponding metaphosphate melts decrease their activation energy.

Figure 2 now shows the activation energy at the high viscosity region as determined from the Arrhenius plots after beam-bending measurements in the same metaphosphate glasses as a function of the modifier field strength. The results of activation energy present a decreasing trend with the IFS of the modifier, being the alkali metaphosphate glasses those with the highest value of activation energy while alkaline-earth compositions show rather similar values. The fact that $\mathrm{Mg}$ and $\mathrm{Zn}$ give rise to smaller viscosity activation energy of their melts causes the increase in their CSA values and this may be interpreted as a consequence of their lower coordination numbers meanwhile, as it can be seen in Fig. 2, the high viscosity range activation energy presents decreasing values according to their field strength. In particular, the much lower activation energy for $\mathrm{Zn}\left(\mathrm{PO}_{3}\right)_{2}$ should also be influenced by the particular electronic structure of $\mathrm{Zn}$ as it does regarding its glass transition temperature, which is much lower than the one of alkaline-earth glasses despite the higher field strength of $\mathrm{Zn}^{2+}$ ions [1]. 
Figure 3 gathers the activation energy of melts viscosity as a function of the CSA of ${ }^{31} \mathrm{P}$, where the inset gathers the CSA values as obtained from the simulation of the ${ }^{31} \mathrm{P}$ NMR static spectra as a function of the IFS values. As it can be seen, the activation energy now slowly changes between $\mathrm{NaPO}_{3}$ and $\mathrm{LiPO}_{3}$, below -133 ppm, while it sharply increases from $\mathrm{Ba}\left(\mathrm{PO}_{3}\right)_{2}$ (-129 ppm) up to $-118 \mathrm{ppm}$ that corresponds to the $\mathrm{Ca}\left(\mathrm{PO}_{3}\right)_{2}$ glass. Alkali metaphosphates with the lowest activation energy result in glasses with bigger CSA while alkaline-earth ones show smaller values of CSA as their viscosity activation energy increases. The calcium metaphosphate composition is now the one that presents the lowest CSA and highest activation energy, as seen in Fig. 1.

\section{Discussion}

As introduced above, the chemical shift anisotropy values in the metaphosphate glasses followed a non-linear trend with the strength of the modifiers. Basically, the CSA of an atom represents a measure of the magnetic anisotropy caused by the chemical environment surrounding the nucleus, which can give rise to anisotropic signals under static conditions in solid state [9]. At the temperatures of the melt, with the lowest viscosities, however, the anisotropy of the phosphorous nuclei would be null, as in liquid state where molecular motions average the anisotropic terms and give rise to isotropic signals only. Therefore, the CSA of the ${ }^{31} \mathrm{P}$ atoms measured by NMR in each glass composition must originate from the structural rearrangements of the network upon cooling the melts. Assuming similar cooling rates under laboratory conditions, each CSA will become different depending on the composition and, most importantly, on the rate of increase of viscosity during cooling, i.e. the activation energy of viscous flow above the liquidus temperature.

According to the model of Hoppe for the structure of phosphate glasses [10], metaphosphate compositions would be built up of by two interpenetrating networks of $\mathrm{PO}_{4}$ and $\mathrm{MO}_{\mathrm{n}}$ 
polyhedra characterized by covalent and ionic bonds, respectively. Furthermore, the formation of enriched regions with higher concentration of modifier atoms where the $\mathrm{MO}_{\mathrm{n}}$ units may share corners, edges and faces can also be described by the Modified Random Network model of Greaves [11]. As seen in Fig. 2, the activation energy at the high viscosity range decreases with the increase of the modifier field strength. We interpret this behavior following the interpretation of Greaves by which "the interfaces between ionic and covalent regions might have lower viscosities". Thus, a higher degree of modified random network is originated in the glasses with higher cationic field strength, where stronger bonds between the modifier atoms and oxygens make phosphorus atoms to keep higher isotropic character upon cooling down the melt (Fig. 3) as a consequence of the higher viscosity activation energy above the liquidus temperature, resulting in a network with higher separation degree of the $\mathrm{PO}_{4}$ and $\mathrm{MO}_{\mathrm{n}}$ enriched regions that give rise to an overall lower viscosity activation energy above the glass $\mathrm{T}_{\mathrm{g}}$.

We do understand that the free activation energy of the viscous flow at the high viscosity range might be different from the apparent activation energy that we are considering here due to a non-negligible entropic term at these temperatures. However, as we pointed above, we already showed that there might be a close relationship between the fragility behavior of the glasses in terms of the ratio between the high and low viscosity $E_{a}$ values following the interpretation of Doremus [12] and their medium range order structure. Therefore, we did not enter into the thermodynamic analysis but just looked at the direct comparison of the measured data. In Fig. 4 the ratio of Doremus, as calculated from the values of activation energy from Fig. 1 and 2, is represented as a function of the IFS of the modifiers. Again, as in Fig. 1, there are two fields with different slopes where the ratio of Doremus decreases with the cation field strength, below and above the calcium composition. Furthermore, a much bigger decrease is observed for low IFS values than for the glasses with higher strength of the 
modifier. There is, of course, a strong influence of the smaller values of the $E_{a}$ at low viscosity for $\mathrm{Mg}$ and $\mathrm{Zn}$ metaphosphates that makes the ratio of Doremus to present higher values than expected from the trend. So, as discussed above, the lower viscosity activation energy of the two last compositions determines network structures showing lower values of the CSA for $\mathrm{Mg}$ and $\mathrm{Zn}$ glasses that are directly reflected onto higher fragility than that expected for their IFS. It can be inferred that the higher the anisotropy of the chemical environment of $\mathrm{PO}_{4}$ tetrahedra in the glass the higher is the fragile character, which is ultimately determined by the rate of increase of viscosity upon cooling and the resultant network structure. The inset in Fig. 4 plots the Doremus ratio vs. the CSA values for the corresponding glasses, and it can be clearly seen that there exists a very regular decreasing behavior of the activation energies ratio and the anisotropy character of phosphorus. Therefore, we propose here a new conceptual definition of the glass fragility as follows: a certain composition will be more fragile when there is greater difference between the structures of the rigid glassy state and that of the liquid in equilibrium from where the former has been obtained.

\section{Conclusions}

Through the relationships between the NMR chemical shift anisotropy of ${ }^{31} \mathrm{P}$, that represents the degree of the isotropic/anisotropic character surrounding the phosphorous nuclei as measured in the glass, and the activation energy for the viscous flow at the high and low viscosity ranges, we conclude that the way the phosphate glass network rearranges itself upon cooling from the melt depends directly on the strength of the bonds between the modifier cations and the oxygens. Therefore, depending on this strength and the rate of viscosity increase upon cooling, the modified random network that is originated for each glass will present different degree of links between subnetworks of ionic and covalent bonds that 
determine the viscosity change over the glass transition, i.e. the glass fragility. As a consequence, a new definition of glass fragility is proposed as by the difference between the structures of the liquid and the glassy state.

\section{Acknowledgements}

Funding from project MAT2013-48246-C2-1-P from MINECO of Spain is acknowledged and the authors are thankful to L. Sánchez-Muñoz from CSIC for the fruitful discussions.

\section{References}

[1] L. Muñoz-Senovilla, F. Muñoz, J. Non-Cryst. Solids 385 (2014) 9.

[2] R.K. Brow, J. Non-Cryst. Solids 263\&264 (2000) 1.

[3] R.K. Brow, J. Am. Ceram. Soc., 74 (6); (1991) 1287.

[4] A.R Grimmer, U. Haubenreisser, Chem. Phys. Lett. 99 (1983) 487.

[5] T.M. Duncan, D.C. Douglass, Chem. Phys. 87 (1984) 339.

[6] L. Muñoz-Senovilla, PhD Thesis, Universidad Autónoma de Madrid (2016).

[7] V.A. Dietzel, Ztschr. Elektrochem. 48(1) (1942) 9.

[8] S. V. Nemilov, J. Non-Cryst. Solids 353 (2007) 4613.

[9] V.I. Bakhmutov, in Practical NMR Relaxation for Chemists, John Wiley \& Sons (2004)

59.

[10] U.W. Hoppe, J. Non-Cryst. Solids 195 (1996) 138.

[11] G.N. Greaves, J. Non-Cryst. Solids 71 (1985) 203.

[12] R.H. Doremus, Am. Ceram. Soc. Bull. 82(3) (2003) 59. 


\section{Figure captions}

Figure 1: Activation energy for the viscous flow at the low viscosity range of the metaphosphate glasses as a function of the ionic field strength of the modifier cation (IFS). The lines are drawn as a guide for the eyes.

Figure 2: Activation energy of the high viscosity range as a function of the modifier field strength. The line is drawn as a guide for the eyes.

Figure 3: Melt viscosity activation energy as a function of the chemical shift anisotropy of the phosphorus atoms as determined through the simulation of the ${ }^{31} \mathrm{P}$ static NMR spectra in the metaphosphate glasses. The inset shows the behavior of the CSA values against the IFS of the modifier cation in the glass composition. Lines are drawn as guide for the eyes.

Figure 4: Doremus ratio between the activation energy of the viscous flow at the high and low viscosity ranges against the modifier field strength and the ${ }^{31} \mathrm{PCSA}$ values (inset). Lines are drawn as guides for the eyes. 


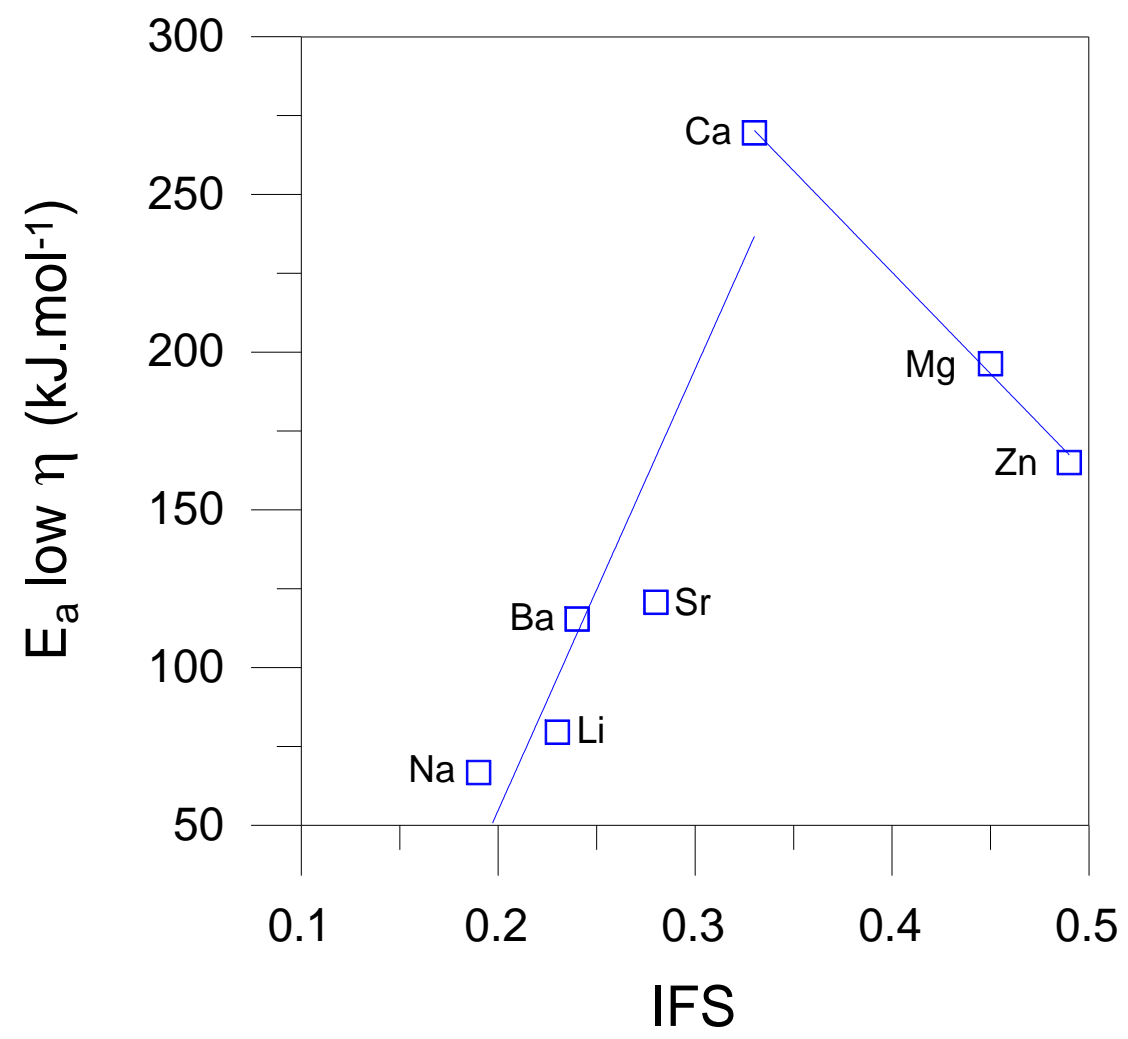

Figure 1 


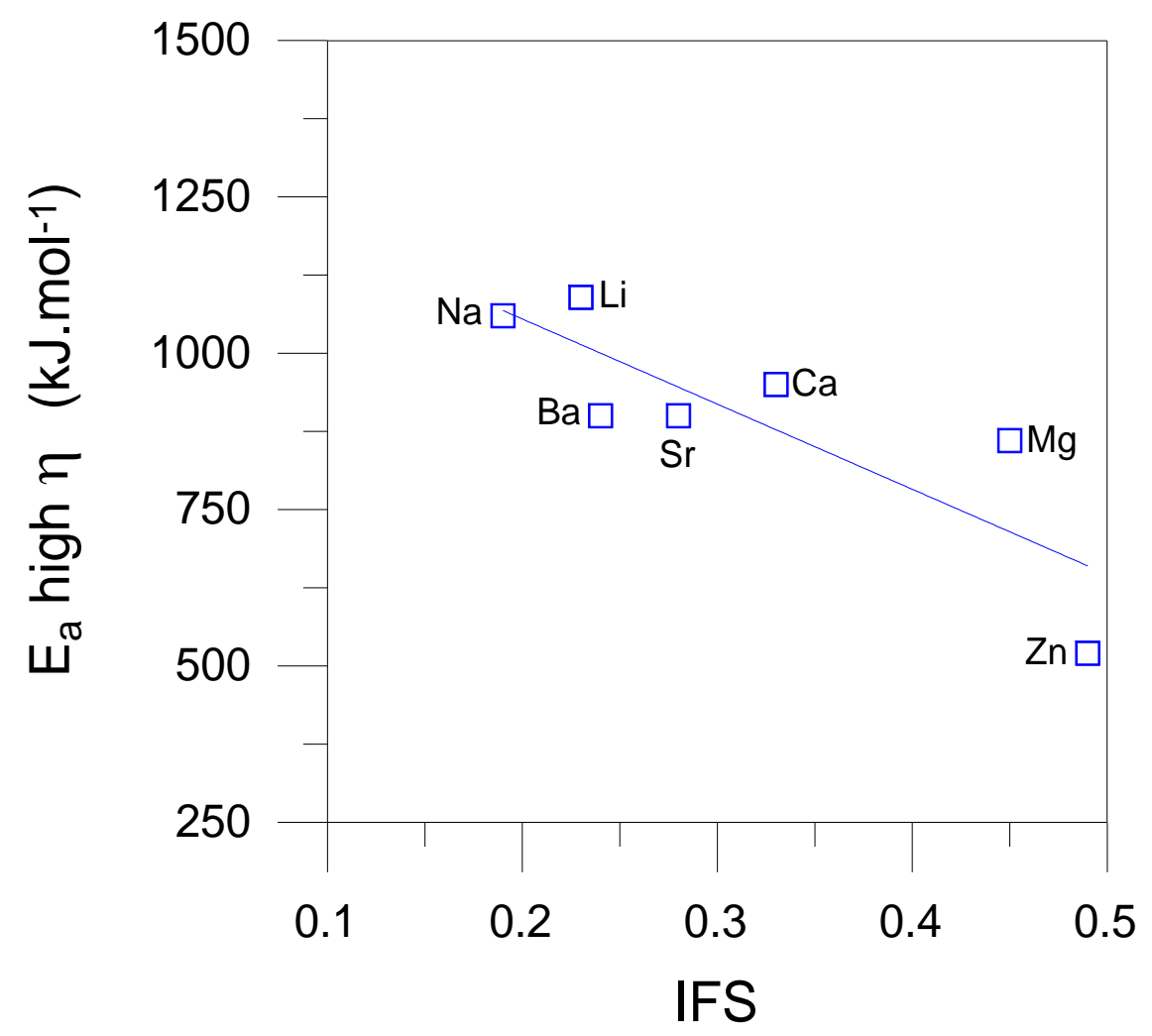

Figure 2 


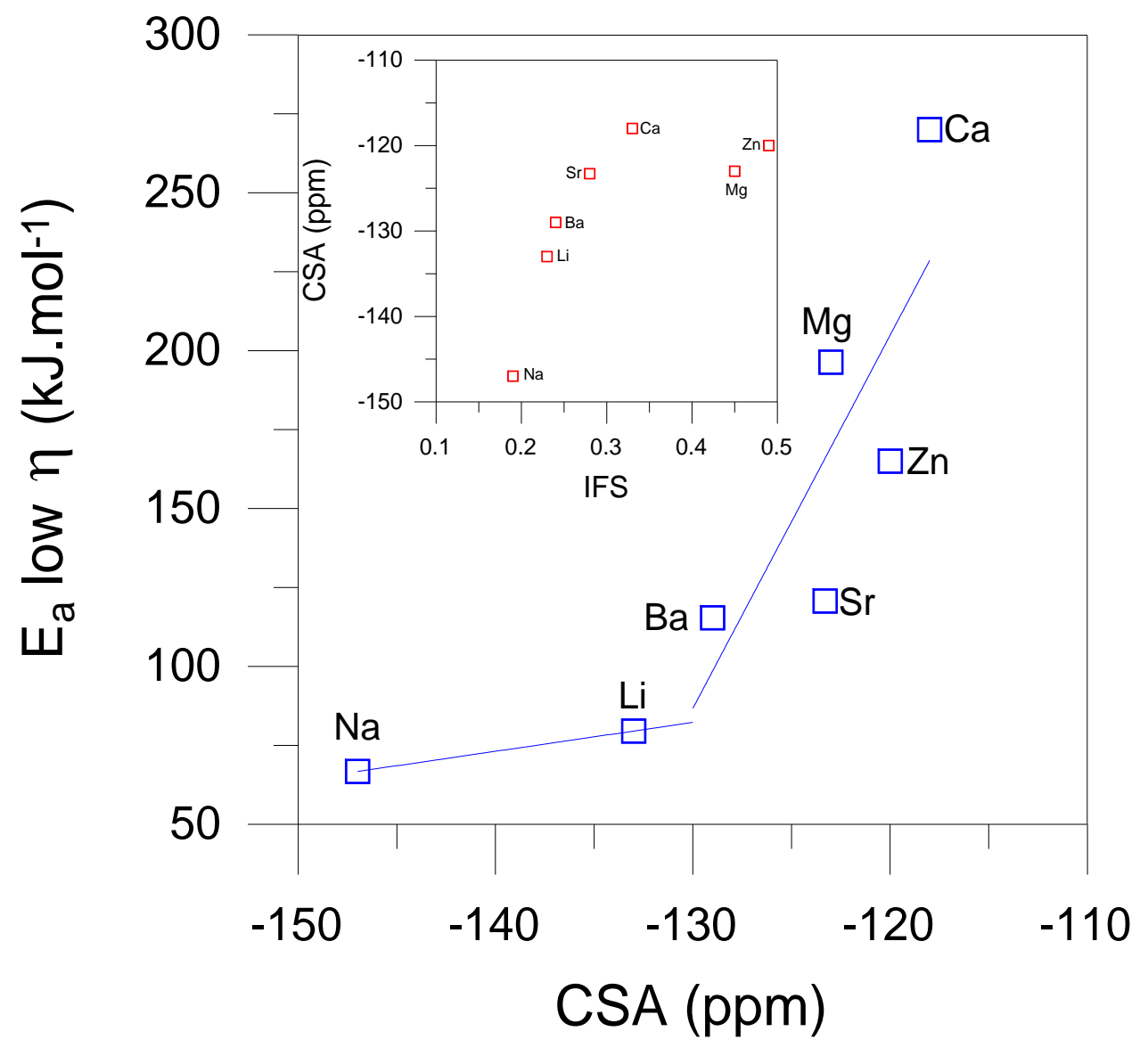

Figure 3 


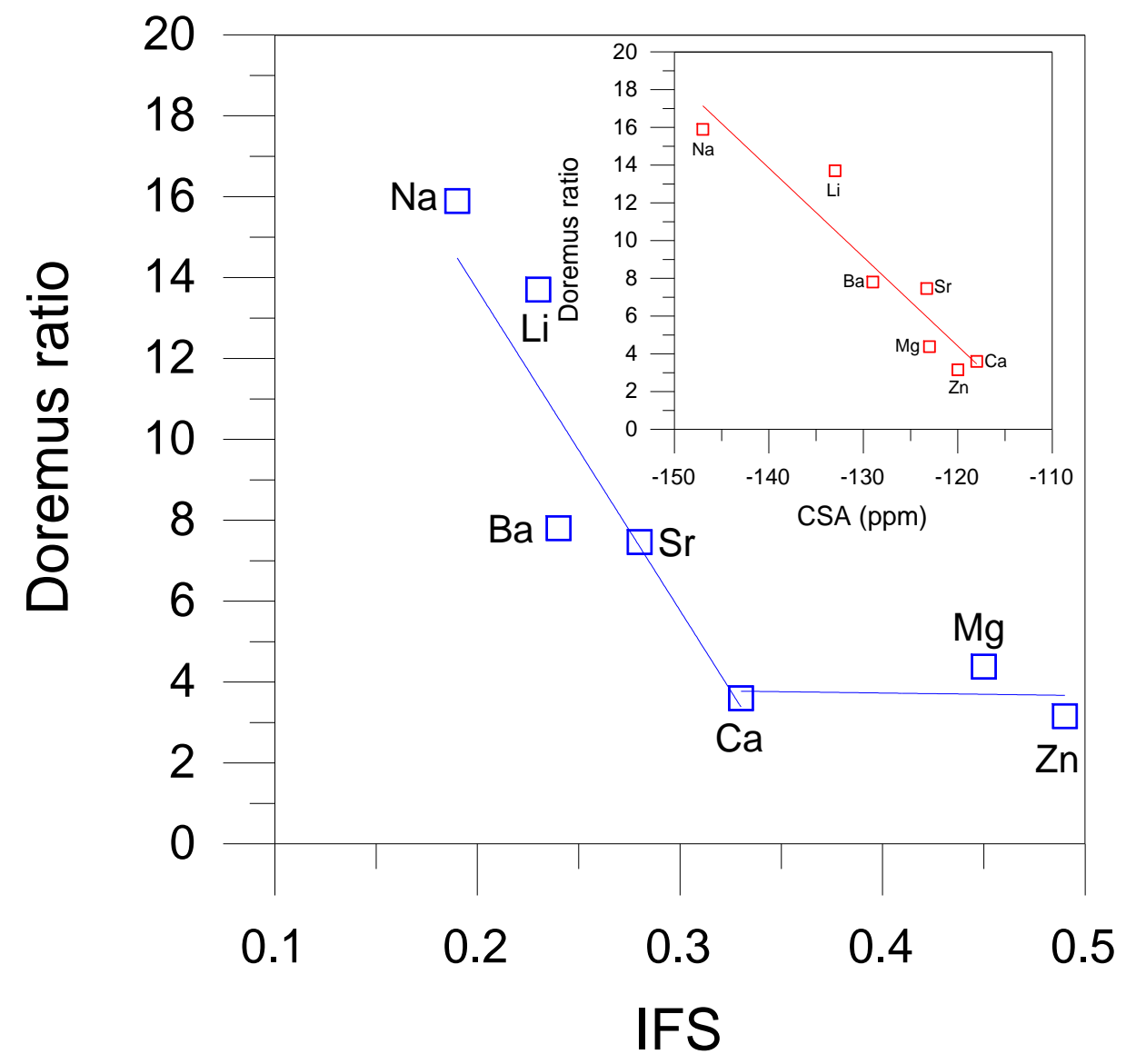

Figure 4 